\title{
Intellectual disability associated with a homozygous missense mutation in THOC6
}

\author{
Chandree L Beaulieu ${ }^{1 \dagger}$, Lijia Huang ${ }^{1 \dagger}$, A Micheil Innes ${ }^{2,3}$, Marie-Andree Akimenko ${ }^{4}$, Erik G Puffenberger ${ }^{5}$, \\ Charles Schwartz ${ }^{6}$, Paul Jerry ${ }^{7}$, Carole Ober ${ }^{8}$, Robert A Hegele ${ }^{9}$, D Ross McLeod ${ }^{2,3}$, Jeremy Schwartzentruber ${ }^{10}$, \\ FORGE Canada Consortium, Jacek Majewski ${ }^{10}$, Dennis E Bulman ${ }^{1}$, Jillian S Parboosingh ${ }^{2,3+}$ and Kym M Boycott ${ }^{1{ }^{*+}}$
}

\begin{abstract}
Background: We recently described a novel autosomal recessive neurodevelopmental disorder with intellectual disability in four patients from two related Hutterite families. Identity-by-descent mapping localized the gene to a $5.1 \mathrm{Mb}$ region at chromosome 16p13.3 containing more than 170 known or predicted genes. The objective of this study was to identify the causative gene for this rare disorder.

Methods and results: Candidate gene sequencing followed by exome sequencing identified a homozygous missense mutation p.Gly46Arg, in THOC6. No other potentially causative coding variants were present within the critical region on chromosome 16. THOC6 is a member of the THO/TREX complex which is involved in coordinating mRNA processing with mRNA export from the nucleus. In situ hybridization showed that thoc 6 is highly expressed in the midbrain and eyes. Cellular localization studies demonstrated that wild-type THOC6 is present within the nucleus as is the case for other THO complex proteins. However, mutant THOC6 was predominantly localized to the cytoplasm, suggesting that the mutant protein is unable to carry out its normal function. siRNA knockdown of THOC6 revealed increased apoptosis in cultured cells.
\end{abstract}

Conclusion: Our findings associate a missense mutation in THOC6 with intellectual disability, suggesting the THO/ TREX complex plays an important role in neurodevelopment.

Keywords: Intellectual disability, THOC6, THO/TREX complex, mRNA export, Hutterite

\section{Background}

Intellectual disability (ID) is the most frequent handicap affecting children and it is one of the greatest challenges in healthcare as ID is associated with life-long impairments that have a profound impact on individuals, families, and society. Genetic causes of ID are diverse and include chromosomal aberrations and autosomal dominant, X-linked, autosomal recessive, and mitochondrial DNA mutations. Nonsyndromic ID is characterized by ID as the sole clinical feature in patients, while syndromic ID occurs in combination with one or more additional clinical features. Recently, next-generation sequencing of 136 consanguineous families identified 23 previously implicated ID genes and 50 novel candidate genes, confirming

\footnotetext{
* Correspondence: kboycott@cheo.on.ca

${ }^{\dagger}$ Equal contributors

${ }^{1}$ Children's Hospital of Eastern Ontario Research Institute, University of Ottawa, 401 Smyth Road, Ottawa, ON K1H 8L1, Canada

Full list of author information is available at the end of the article
}

the suspected significant genetic heterogeneity underlying ID [1].

The Hutterites are a German-speaking Anabaptist group that arose during the Protestant Reformation (1528) in South Tyrol (Austria) [2]. The Hutterite population has been living on the North American prairies since the late 1800 s, now numbers over 40,000 , and is comprised of three essentially endogamous groups, Schmiedeleut, Dariusleut, and Lehrerleut. Their genetic isolation, small founder population, excellent genealogical records, large completed family size, and uptake of modern health care facilitates genetic studies [2]. Over 30 autosomal recessive conditions have been identified within this population and additional novel Mendelian disorders continue to be recognized [3].

We recently described a novel autosomal recessive ID disorder in two sets of sisters from related Dariusleut Hutterite families [4]. As previously described, their

\section{Biomed Central}


clinical features include significant learning disabilities and head circumference at the 2nd centile without apparent structural CNS malformations on MRI. All four patients share recognizable facial features including a tall forehead with high anterior hairline, deeply-set eyes with short, upslanting palpebral fissures, long nose with lowhanging columella, and thick vermilion of the upper and lower lip. Other clinical features include dental malocclusion and caries, myopia, malformations of the heart (one patient with patent ductus arteriosus and ventricular septal defect, another with ventricular septal defect only), and renal abnormalities (one patient with horseshoe kidney, another patient with left renal agenesis and renal failure identified at age 13 requiring dialysis followed by transplant at age 15 years; at diagnosis the unilateral right kidney was echogenic and atrophic on ultrasound imaging) [4]. The patients underwent neuropsychological testing to further characterize their learning disabilities and the results were consistent with moderate ID without a significant difference between verbal comprehension and perceptual reasoning. Based on the clinical presentation, this disorder is best classified as a syndromic form of ID, but due to the subtle and variable nature of the additional features the diagnosis of syndromic ID could be easily missed. Furthermore, the distinction between syndromic and nonsyndromic is becoming blurred with a subset of ID genes causing both forms of disability $[5,6]$.

A single region on $16 \mathrm{p} 13.3$ was identified by genomewide homozygosity mapping with DNA samples from the four patients using a 50K Affymetrix GeneChip SNP array followed by refinement with microsatellite markers using all available family members [4]. The maximum size of the region was $5.1 \mathrm{Mb}$ with the distal boundary at $1,404,019$ and the proximal boundary at $6,458,669$ (NCBI Build 36.3) [4]. Sanger followed by exome sequencing identified a single potential disease-causing missense mutation in THOC6 (fSAP35; WDR58). THOC6 is a part of the THO complex which is involved in coordinating mRNA processing with export. We demonstrate that the p.Gly46Arg substitution results in protein mislocalization to the cytoplasm suggesting that the mutant protein is unable to carry out an export function. Moreover, depletion of THOC6 induces apoptosis in mammalian cells. These findings indicate that the THOC6 missense mutation perturbs protein function, supporting a disease-association between THOC6 and intellectual disability and an important role for the $\mathrm{THO} /$ TREX complex in neurodevelopment.

\section{Methods}

\section{Patient recruitment}

Institutional Research Ethics Board approval for the study reported here was obtained from the University of Calgary and the Children's Hospital of Eastern Ontario and informed consent was obtained from responsible persons (parents) on behalf of all study participants. Total genomic DNA was extracted from blood following standard procedures.

\section{Sanger sequencing}

Ninety-seven out of the 173 genes within the $5.1 \mathrm{Mb}$ critical region on 16p13.3 (NCBI build 36.3, including hypothetical genes and pseudogenes) were PCR amplified and Sanger sequenced. These genes were chosen based on expression or function indicating a potential role in neurodevelopment; genes associated with dissimilar disorders or unrelated functions were excluded from sequencing. Primers were designed to assess the coding regions and intron-exon boundaries of the prioritized genes using the program Oligo (Molecular Biology Insights, Inc., Cascade, CO). PCR and bidirectional sequencing was performed on DNA samples from a patient, a parent, and unaffected control. Primer sequences and reaction conditions are available upon request. Sequence subtraction analysis was performed using Mutation Surveyor (SoftGenetics LLC, State College, PA).

\section{Exome sequencing}

Exome capture and high-throughput sequencing of DNA from one patient was performed at the McGill University and Genome Québec Innovation Centre (Montréal, Canada). Exome target enrichment was performed using the Agilent SureSelect $50 \mathrm{Mb}$ All Exon Kit (V3), and sequencing (Illumina HiSeq) generated 14 Gbp of $100 \mathrm{bp}$ paired-end reads. An in-house annotation pipeline was used to call and annotate coding and splice-site variants. Reads were aligned to hg19 using BWA [7] and duplicate reads were marked using Picard [8] and excluded. Single nucleotide variants and short insertions and deletions (indels) were called using SAMtools mpileup [9] and bcftools and quality-filtered to require a minimum $20 \%$ of reads supporting the variant call. Variants were annotated using Annovar [10] as well as custom scripts to select coding and splice-site variants, and to exclude common $(\geq 1 \%$ minor allele frequency) polymorphisms represented in the NHLBI exome server [11], or in 435 control exomes sequenced at the McGill University and Genome Québec Innovation Centre. Variants within the mapped region at 16 p13.3 were considered as candidates. Coverage of genes within the region was assessed to determine the fraction of bases in each exon with at least 5 reads covering the position. Although average coverage was much higher, $5 \times$ was deemed sufficient to call variants within this homozygous region. To determine the proportion of CCDS exons with sufficient coverage, exons of all isoforms within the region were counted (exons present in multiple isoforms were counted only once) and exons with less than $99 \%$ coverage at $5 \times$ were deemed incomplete. 


\section{Taqman genotyping}

500 Schemiedeleut controls, 92 Daruisleut controls, and 120 Lehrerleut controls were genotyped by a TaqMan SNP genotyping assay for the variant. Life Technologies' TaqMan genotyping protocol and mix were used following standard procedures (Forward Primer: AGAAACTTCCCACATGGT GAGAC, Reverse Primer: ATTAGCCCTGGCACTTGGC, Wild-type Probe: VIC-TG GCAACAATTACGGGC-mgb (minor groove binder), Mutant Probe: FAM-ACAATTA CAGG CAGATT-mgb).

\section{Plasmids and site-directed mutagenesis}

Wild-type (WT) THOC6 cDNA was purchased from the Centre for Applied Genomics (Toronto). The primer sets: 5' TTA GGA TCC ATG GAC TAC AAG GAT GAC GAT GAC AAG GAG CGA GCT GTG CCG CTC 3’/5' GTA GCG GCC GC TCA GAA GGA CAG GGA GAA GGC TCG 3' and 5' TTA GGA TCC ATG GAG CGA GCT GTG CCG CTC 3'/5' GGA GCG GCC GC TCA CTT GTC ATC GTC ATC CTT GTA GTC GAA GGA CAG GGA GAA GGC TCG 3' were used to PCR amplify the full length THOC6 cDNA in order to introduce a FLAG tag at the $\mathrm{N}$ - and $\mathrm{C}$-terminus, respectively and subsequently the cDNA was subcloned into pcDNA3. Site-directed mutagenesis of THOC6 was performed using the QuikChange II mutagenesis kit (Agilent). The mutated cDNA constructs were sequenced to confirm the fidelity of the mutagenesis reactions.

\section{Immunostaining}

HeLa cells were seeded at $2 \times 10^{5}$ cells/well on glass coverslips in six-well plates and transiently transfected with FLAG-tagged WT THOC6 or mutant THOC6 plasmids with lipofectamine 2000 (Invitrogen). Twenty-four hours after the transfection, the cells were fixed in $2 \%$ paraformaldehyde and permeabilized with PBS containing TritonX-100 (0.05\%). Cells were incubated with anti-FLAG antibody (Sigma) for $1 \mathrm{~h}$ followed by incubation with Alexa 488-conjugated goat anti-mouse IgG secondary antibody (Invitrogen). The cells were stained with DAPI and mounted onto microscope slides. Images were obtained with a Nikon Eclipse TE2000-E system controlled and processed by EZ-C1 3.50 (Nikon) software. Two replicates and a chi-square test were performed on each replicate.

\section{siRNA transfection}

Human THOC6-specific and non-targeting control siRNAs were purchased from Dharmacon. HeLa cells were plated at a density of $2 \times 10^{5}$ cells/well on a coverslip in a six-well plate, $24 \mathrm{~h}$ prior to transfections. Cells were transfected with $100 \mathrm{nM}$ of THOC6-specific or nontargeting scramble siRNA with Lipofectamine RNAiMAX (Invitrogen) and cultured for $48 \mathrm{~h}$.

\section{TUNEL staining}

TUNEL staining was performed using the in situ cell death detection kit, TMR red (Roche), following the manufacturer's protocol. Two replicates and a chi-square test were performed on each replicate.

\section{Zebrafish whole-mount in situ hybridization}

Whole-mount in situ hybridization was performed on embryos at 24, 48, and $72 \mathrm{~h}$ post fertilization (hpf) as described previously [12]. Briefly, full-length cDNA of zebrafish thoc6 was obtained from Open Biosystems (EDR5649-100965848). The cDNA was cloned into pGEM-T vector (Promega) and used as a template for in vitro synthesis of an antisense mRNA probe. Embryos were fixed in $4 \%$ paraformaldehyde in PBS, hybridized with DIG-labeled riboprobes at $65^{\circ} \mathrm{C}$, and followed by incubation with anti-DIG antibody conjugated with alkaline phosphatase (AP) (Roche). NBT and BICP were used as the substrates of AP to generate the purple coloration.

\section{Results and discussion}

\section{Identification of THOC6 as candidate gene for ID}

Prior to the availability of whole-exome sequencing, the coding regions of 97 out of the 173 genes within the mapped region (NCBI build 36.3, including hypothetical genes and pseudogenes) were Sanger sequenced in a patient and parent. These genes were chosen based on expression or function indicating a potential role in neurodevelopment; genes associated with dissimilar disorders or unrelated functions were excluded from sequencing. A potential disease-causing missense mutation was identified in THOC6 (fSAP35), c.136G>A (p.Gly46Arg) (RefSeq NM_024339.3) (Figure 1A). Sanger sequencing confirmed the presence of this potential mutation in the homozygous state in the other three patients.

Exome sequencing was performed to determine if any additional potentially pathogenic variants were present in the coding sequence of genes within the critical region. The mean exome read depth for the sample was 143x. Exome sequencing coverage was determined for all CCDS annotated genes (hg19) within the region and $92 \%$ of exons from all isoforms were covered completely at greater than $5 \times$. When Sanger sequencing results were included this increased to $96 \%$ of exons being covered. Gene coverage statistics are included in Additional file 1: Table S1. Variants identified by exome sequencing were filtered to exclude common $(\geq 1 \%$ minor allele frequency) polymorphisms represented in the NHLBI exome server [11], dbSNP, or in 435 control exomes sequenced at our center. The only rare variant in the mapped region was the THOC6 homozygous variant c.136G >A, which was not present in any of the controls. 


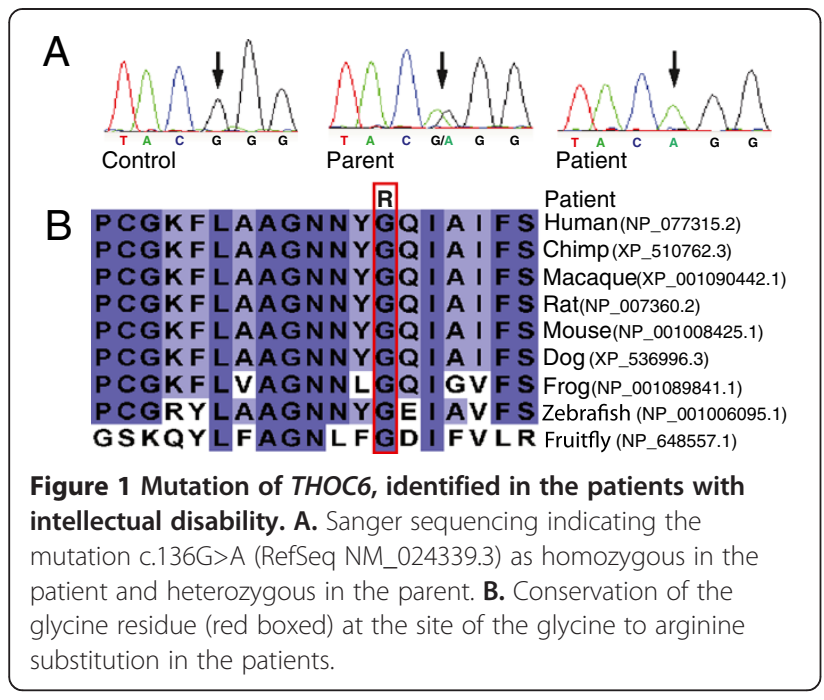

The c.136G $>$ A variant was found in the heterozygous state in the parents of the patients and none of the six unaffected siblings available for testing were homozygous. The variant was not seen in 150 controls from the general population. Frequency of the variant was determined in the three Hutterite leuts by Taqman genotyping. The variant was seen at a frequency of $3 \%$ in 92 Dariusleut controls and at a frequency of $2 \%$ in 120 Lehrerleut controls; no homozygous controls were identified. The variant was not seen in 500 Schmiedeleut controls indicating it is likely shared only between the Dariusleut and Lehrerleut and that additional affected individuals may exist within these communities. The glycine at position 46 is highly conserved between species and occurs within a relatively conserved region (Figure 1B), suggesting that this amino acid plays an important role in THOC6. Polyphen [13] and SIFT [14] predicted THOC6 p.Gly46Arg to be damaging.

Next, we sequenced THOC6 in a collection of 140 female patients with ID and microcephaly; a female patient cohort was used to enrich for non X-linked ID. No rare homozygous or compound heterozygous variants considered likely to be disease-causing were identified.

THOC6 is a part of the THO complex which is involved in coordinating mRNA processing with export. In humans, this complex is comprised of THOC1, THOC2, THOC5, THOC6, and THOC7 [15]. The THO complex components also interact with UAP56, ALY, and TEX forming the larger TREX complex (transcription export complex) [15-17]. In yeast, this complex was found to have a role in coupling transcription and polyadenylation to mRNA export with mutants showing defects in transcription, polyadenylation, nuclear accumulation of poly (A) RNA, the formation of heavy chromatin, and accumulation of stalled nuclear pore components [17-19]. The necessity of the THO complex for export of individual transcripts in yeast has been linked to genes with strong promoters and rapid transcription rates [20,21]. Initial studies in human cell lines found THOC1 to bind DNA and interact with RNA Polymerase II as in yeast [22]; however, recent literature has favoured the hypothesis that in metazoan organisms the THO complex couples mRNA processing with export. In metazoans, the THO complex components have only been found bound to processed transcripts [15] and export of transcripts to the cytoplasm by $\mathrm{THO}$ was determined to be both cap and splicing dependent [23].

\section{Impaired cellular localization of mutant THOC6 p.Gly46Arg}

Members of the THO complex including THOC1, THOC2, THOC5, and THOC7 have been found to be localized to the nucleus and to shuttle between the nucleus and the cytoplasm [24-26]. Within the nucleus, they have been found to co-localize with splicing factors in nuclear speckle domains and function in the release of mRNA from these domains [15,27]. We subcloned the WT and mutant THOC6 (c.136G>A) cDNA with a FLAG epitope at the C-terminus into pcDNA3 vector. Immunostaining was performed with anti-FLAG antibody on HeLa cells transfected with the WT and mutant constructs. The majority of cells showed a speckled nuclear localization of WT THOC6 protein similar to what has been seen for THOC1 and THOC2 [15,22,28] (Figure 2A), whereas the mutant THOC6 p.Gly46Arg protein was confined to the cytoplasm (Figure 2B). Similar results were obtained using N-terminal FLAG tagged proteins. The difference in localization was significant for both the C-terminal and N-terminal FLAG tagged protein (chi-square test) (Additional file 2: Figure S1). The absence of localization of the p.Gly46Arg mutant protein in the nucleus suggests that the mutant protein's normal function may be perturbed. In HeLa cells, knockdown of THOC2, THOC1, and THOC7 led to polyA RNA nuclear accumulation, whereas knockdown of THOC5, THOC6, and TEX did not [29]. Knockdown of the THO complex in Drosophila appears to affect the export of only a very small subset of mRNA transcripts including the rapidly transcribed inducible HSP7O transcripts [16]. Knockdown of THOC5 and THOC6 was seen to lead to the retention of HSP70 mRNA in the nucleus after heat shock [24]. Further elucidation of the role of THOC6 in mRNA export will provide important insights into the pathophysiology of this disorder.

\section{Depletion of THOC6 increases apoptosis in mammalian cells}

Apoptosis occurs during brain development and is highly regulated; animal models and human disorders suggest that increased levels of apoptosis can lead to 


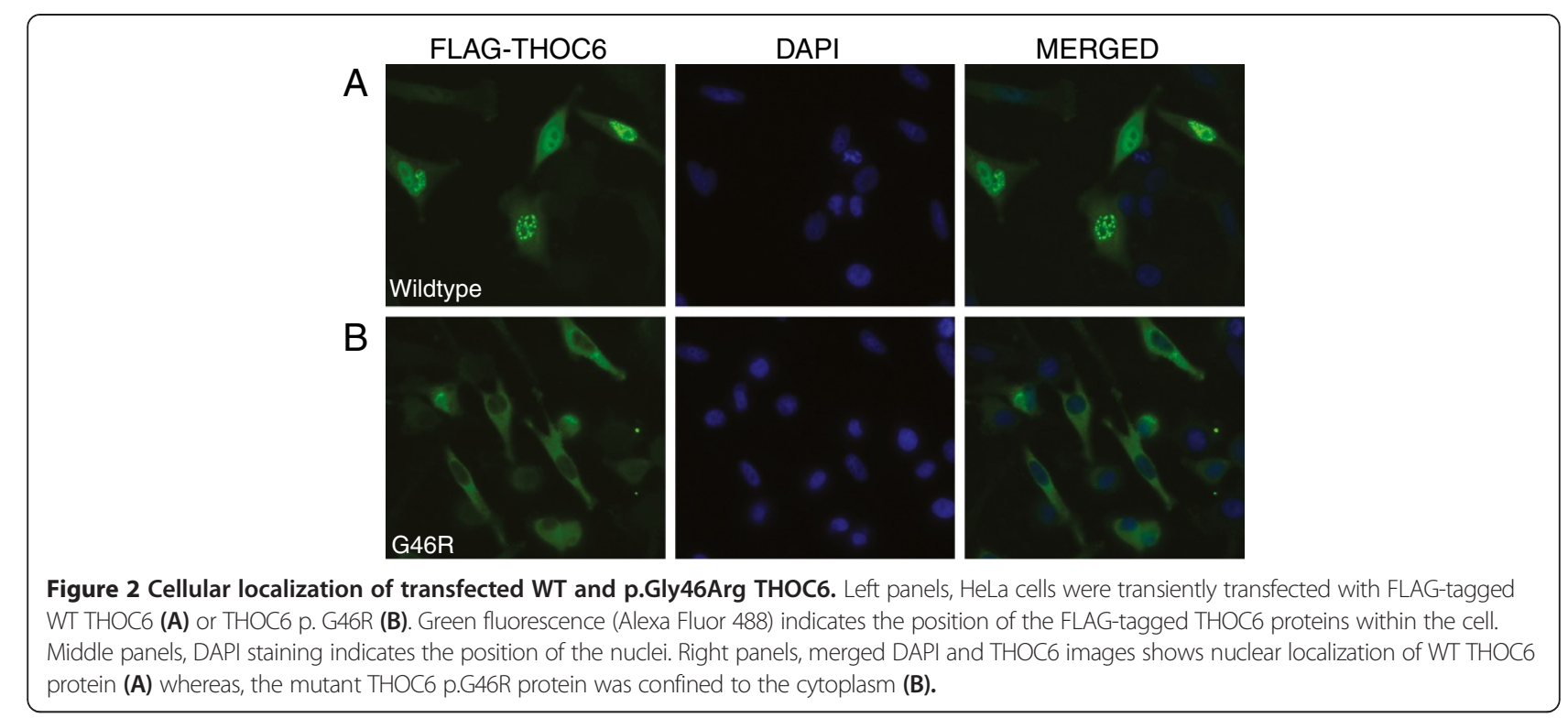

severe neurological defects including microcephaly [30-32]. There has been evidence suggesting a role of the THO complex in the survival of rapidly proliferating cells by preventing apoptosis $[33,34]$. We sought to determine whether loss of THOC6 expression causes apoptosis in a mammalian cell line. THOC6 gene-specific siRNAs were used to knockdown THOC6 in HeLa cells. A robust decrease in the levels of THOC6 protein in HeLa cells transfected with THOC6 gene-specific siRNAs (Additional file 3: Figure S2A) was observed. TUNEL staining was performed to examine the level of apoptosis. A significantly increased proportion of cells with positive TUNEL staining was seen in cells transfected with THOC6 siRNAs, compared to the cells transfected with a control siRNA (Figure 3 and Additional file 3: Figure S2B), indicating that loss of THOC6 leads to an increase in apoptosis.
Expression of Thoc6 in the central nervous system during zebrafish embryonic development

Genes implicated in neurodevelopment and ID are diverse in function and many are not limited to central nervous system expression, but detailed expression studies of THOC6 had not yet been performed. We examined expression of the zebrafish thoc6 ortholog during embryonic development. Zebrafish Thoc6 shares 59\% protein sequence identity with human THOC6, and the p.Gly46 in humans is conserved in the zebrafish sequence. In situ hybridization revealed that thoc6 mRNA is highly expressed in the developing midbrain and the eyes at $24 \mathrm{~h}$ post fertilization (hpf) and the expression becomes restricted afterwards to the posterior part of the midbrain and the midbrain-hindbrain boundary (Figure 4). This expression pattern implicates an important role for THOC6 in neurodevelopment, which is of interest

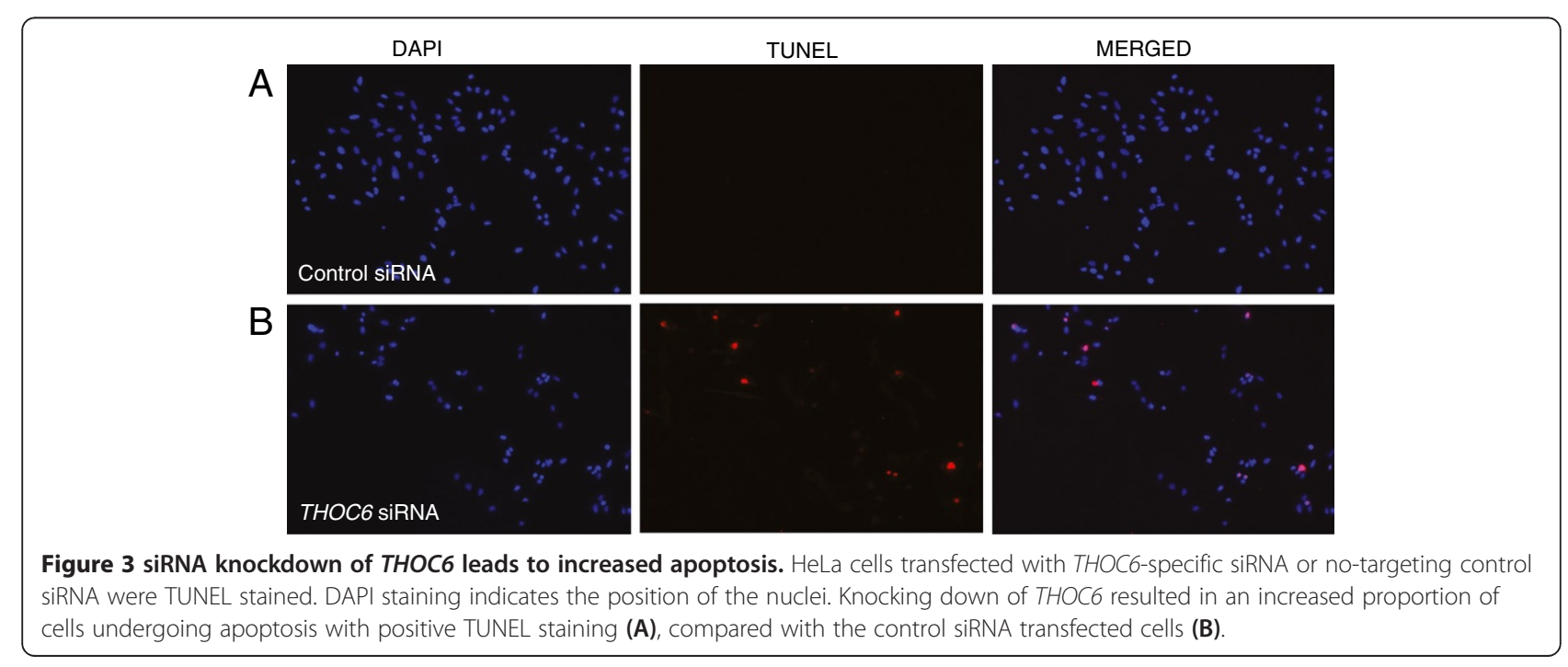




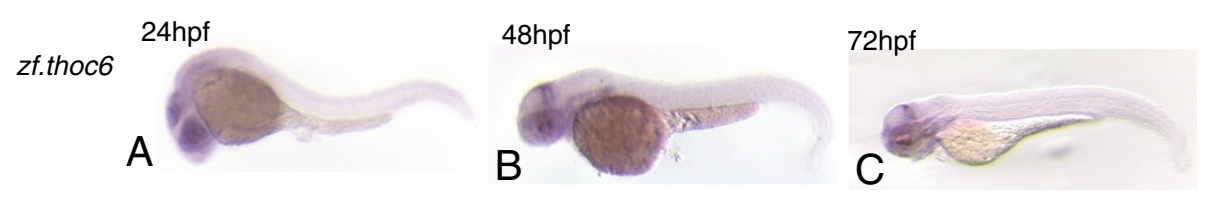

Figure 4 Expression of zebrafish thoc6 in the brain. Expression of zebrafish thoc6 was assessed by whole mount in situ hybridization. Side views of whole mount thoc6 expression observed in 24, 48 and 72 hpf WT embryos. thoc6 is highly expressed in the developing optic tectum and the eyes at $24 \mathrm{hpf}(\mathbf{A})$. This expression decreases at 48 (B) and $72 \mathrm{hpf}$ (C) but a low level of expression is observed at the

midbrain-hindbrain boundary.

considering the central clinical manifestation in the patients is intellectual disability. Given the other, more variable, clinical manifestations observed in the patients, it is likely that THOC6 also has a role in the development of other systems, particularly the heart and kidney.

Well-characterized causes of non-syndromic ID include genes encoding for synaptic proteins, neuronal specific proteins, and those involved in neurotransmitter release [5]. Other causes of ID are not neural specific genes, but genes coding for proteins that participate in defined processes known to be important for brain function such as metabolism and cell adhesion [5,35-37]. Other identified pathways include the Rho GTPases that play a role in regulating the actin cytoskeleton [38], the ERK/MAPK pathway that responds to growth factors [5], and the NF- $\mathrm{KB}$ transcription regulation pathway [39-41]. Genes causing microcephaly with ID include centrosomal, cell cycle, and DNA damage repair genes $[42,43]$. Other ID genes appear to function in fundamental cellular processes, yet give rise to disorders where the predominant or only feature is ID [1,44]; this includes genes involved in mRNA processing such as ZC3H14 [45]. THOC6, as part of a pathway involved in mRNA export and protection against apoptosis, is best classified with this latter group of genes implicated in fundamental cellular processes, though in keeping with the predominant feature of this syndrome, the highest level of expression of THOC6 is in the developing brain.

\section{Conclusion}

In the current study, we have shown that a mutation resulting in THOC6 loss-of-function is associated with a syndromic form of autosomal recessive ID in the Hutterite population. The p.Gly46Arg substitution results in protein mislocalization to the cytoplasm. Moreover, depletion of THOC6 induces apoptosis in mammalian cells. In zebrafish, thoc6 mRNA is highly expressed in the developing central nervous system during embryonic development. Collectively, these findings indicate that THOC6 plays an important role in human neurodevelopment. Given that THOC6 is a member of the THO complex, mutations in other complex members may explain a portion of intellectual disability.

\section{Web resources}

NHLBI Exome Variant Server http://evs.gs.washington. edu/EVS/

Picard http://picard.sourceforge.net/

SAMtools http://samtools.sourceforge.net/

UCSC Genome Browser http://genome.ucsc.edu/cgi-bin/

hgGateway/

SIFT http://sift.bii.a-star.edu.sg/

PolyPhen-2 http://genetics.bwh.harvard.edu/pph2/

\section{Additional files}

Additional file 1: Table S1. Coverage of genes within the mapped region by exome and Sanger sequencing. CCDS genes within the mapped region (including multiple gene isoforms) are listed indicating mean coverage by exome sequencing, percentage of bases covered greater than $5 x$ by exome sequencing, and whether the gene was Sanger sequenced.

Additional file 2: Figure S1. THOC6 p.Gly46Arg mutation leads to statistically significant localization change compared to WT in transfected cells. Cells were classified into categories based on predominant area of THOC6 localization: nuclear localization, cytoplasmic localization, and presence in both the nucleus and cytoplasm. Sample sizes were WT C-FLAG $n=216, p . G 46 R$ C-FLAG $n=206$, WT N-FLAG $n=272$, p.G46R N-FLAG $n=242$, and the Chi-Square test indicated a significant difference $(p<0.0001)$.

Additional file 3: Figure S2. siRNA knockdown of THOC6 leads to statistically significant increase in apoptosis in HeLa cells. A. A robust decrease in the levels of THOC6 protein in HeLa cells transfected with THOC6-specific siRNAs was seen. B. Comparison of apoptosis between control siRNA and THOC6 siRNA transfected cells. Cells that were positive for apoptosis based on TUNEL staining were counted $(n=2500) .5 .7 \%$ of the THOC6 siRNA transfected cells were TUNEL positive compared to $0.7 \%$ of cells transfected with control siRNA. The Chi-Square test indicated this to be a significant increase $(p<0.0001)$.

\section{Abbreviations}

ID: Intellectual disability; Mgb: Minor groove binder; THOC6: THO complex 6 homolog; TREX Complex: Transcription export complex; CCDS: Consensus CDS; SIFT: Sorting intolerant from tolerant;

PolyPhen-2: Polymorphism phenotyping; WT: Wild-type.

\section{Competing interests}

The authors declare that they have no competing interests.

\section{Authors' contributions}

$C L B, L H, D E B, A M I, K M B$, and JSP designed the study. CLB performed Sanger sequencing. JS and JM carried out the analysis of the next-generation sequencing data. RAH, CO, and EGP performed genotyping. CLB and LH performed mammalian cell and zebrafish experiments. JSP supervised Sanger sequencing, MAA supervised zebrafish experiments, DEB supervised mammalian cell experiments, and KMB coordinated all components. CS provided cohort of ID patients. AMI and DRM provided clinical examinations 
and PJ provided subspecialist consultation services. CLB and LH wrote the manuscript and all authors read and approved the manuscript.

\section{Acknowledgements}

We would like to thank the patients and their families for participation. We thank Jackie Morris and Carol Farr for clinical support and Dr. Amanda Smith and Jing Zhang for technical support. We thank Dr. Robin Reed for helpful discussions on the THO/TREX complex. The authors wish to acknowledge the contribution of the high throughput sequencing platform of the McGill University and Génome Québec Innovation Centre, Montréal, Canada. This work was supported in part by research grant \#5-FY09-529 from the March of Dimes Foundation and by the Government of Canada through the FORGE (Finding of Rare Disease Genes) Canada Consortium funded by Genome Canada, the Canadian Institutes of Health Research and the Ontario Genomics Institute (OGI-049). Additional funding to FORGE was provided by Genome Quebec, Genome British Columbia, and the McLaughlin Centre. C.S. received funding from the South Carolina Department of Disabilities and Special Needs. J.S.P. received funding from Alberta Children's Hospital Foundation. K.M.B. is supported by a Clinical Investigatorship Award from the Canadian Institutes of Health Research, Institute of Genetics. This work was selected by the FORGE Canada Consortium Steering Committee: Kym Boycott (leader; University of Ottawa), Jan Friedman (co-lead; University of British Columbia), Jacques Michaud (co-lead; Université de Montréal), Francois Bernier (University of Calgary), Michael Brudno (University of Toronto), Bridget Fernandez (Memorial University), Bartha Knoppers (McGill University), Mark Samuels (Université de Montréal), Steve Scherer (University of Toronto).

\section{Author details}

'Children's Hospital of Eastern Ontario Research Institute, University of Ottawa, 401 Smyth Road, Ottawa, ON K1H 8L1, Canada. ${ }^{2}$ Department of Medical Genetics, University of Calgary, Calgary, AB, Canada. ${ }^{3}$ Alberta Children's Hospital Research Institute for Child and Maternal Health, Calgary, $\mathrm{AB}$, Canada. ${ }^{4}$ Department of Biology, University of Ottawa, Ottawa, ON, Canada. ${ }^{5}$ Clinic for Special Children, Strasburg, PA, USA. ${ }^{6}$ Greenwood Genetic Center, Greenwood, SC, USA. 'Paul Jerry Consulting Psychology, Inc. Medicine Hat, AB, Canada. ${ }^{8}$ Department of Human Genetics, University of Chicago, Chicago, IL, USA. ${ }^{9}$ Robarts Research Institute and University of Western Ontario, London, ON, Canada. ${ }^{10}$ McGill University and Genome Quebec Innovation Centre, Montreal, QC, Canada.

\section{Received: 12 February 2013 Accepted: 16 April 2013}

Published: 26 April 2013

\section{References}

1. Najmabadi H, Hu H, Garshasbi M, Zemojtel T, Abedini SS, Chen W, Hosseini $M$, Behjati F, Haas S, Jamali P: Deep sequencing reveals 50 novel genes for recessive cognitive disorders. Nature 2011, 478:57-63.

2. Hostetler JA, Opitz JM, Reynolds JF: History and relevance of the Hutterite population for genetic studies. Am J Med Genet 1985, 22:453-462.

3. Boycott KM, Parboosingh JS, Chodirker BN, Lowry RB, McLeod DR, Morris J, Greenberg CR, Chudley AE, Bernier FP, Midgley J, et al: Clinical genetics and the Hutterite population: a review of Mendelian disorders. Am J Med Genet Part A 2008, 146A:1088-1098.

4. Boycott KM, Beaulieu C, Puffenberger EG, McLeod DR, Parboosingh JS, Innes AM: A novel autosomal recessive malformation syndrome associated with developmental delay and distinctive facies maps to 16 ptel in the Hutterite population. Am J Med Genet Part A 2010, 152A:1349-1356.

5. Kaufman $L$, Ayub M, Vincent JB: The genetic basis of non-syndromic intellectual disability: a review. J Neurodev Disord 2010, 2:182-209.

6. Frints S, Froyen G, Marynen P, Fryns J: X linked mental retardation vanishing boundaries between non specific (MRX) and syndromic (MRXS) forms. Clin Genet 2002, 62:423-432.

7. Li H, Durbin R: Fast and accurate short read alignment with burrowswheeler transform. Bioinformatics 2009, 25:1754-1760.

8. Picard. http://picard.sourceforge.net.

9. Li H, Handsaker B, Wysoker A, Fennell T, Ruan J, Homer N, Marth G, Abecasis G, Durbin R: The sequence alignment/map format and SAMtools. Bioinformatics 2009, 25:2078-2079.

10. Wang K, Li M, Hakonarson H: ANNOVAR: functional annotation of genetic variants from high-throughput sequencing data. Nucleic Acids Res 2010, 38:e164.

11. NHLBl exome variant server. http://evs.gs.washington.edu/EVS/.
12. Thisse $C$, Thisse B: High-resolution in situ hybridization to whole-mount zebrafish embryos. Nat Protocols 2007, 3:59-69.

13. Adzhubei IA, Schmidt S, Peshkin L, Ramensky VE, Gerasimova A, Bork P, Kondrashov AS, Sunyaev SR: A method and server for predicting damaging missense mutations. Nat Methods 2010, 7:248-249.

14. Kumar P, Henikoff S, Ng PC: Predicting the effects of coding nonsynonymous variants on protein function using the SIFT algorithm. Nat Protocols 2009, 4:1073-1081.

15. Masuda S, Das R, Cheng H, Hurt E, Dorman N, Reed R: Recruitment of the human TREX complex to mRNA during splicing. Genes Dev 2005, 19:1512-1517.

16. Rehwinkel J, Herold A, Gari K, Köcher T, Rode M, Ciccarelli FL, Wilm M, Izaurralde E: Genome-wide analysis of mRNAs regulated by the THO complex in Drosophila melanogaster. Nat Struct Mol Biol 2004, 11:558-566.

17. Sträßer K, Masuda S, Mason P, Pfannstiel J, Oppizzi M, Rodriguez-Navarro S, Rondón AG, Aguilera A, Struhl K, Reed R: TREX is a conserved complex coupling transcription with messenger RNA export. Nature 2002, 417:304-308.

18. Chávez S, Beilharz T, Rondón AG, Erdjument-Bromage H, Tempst P, Svejstrup JQ, Lithgow T, Aguilera A: A protein complex containing Tho2, Hpr1, Mft1 and a novel protein, Thp2, connects transcription elongation with mitotic recombination in Saccharomyces cerevisiae. EMBO J 2000, 19:5824-5834

19. Rougemaille M, Dieppois G, Kisseleva-Romanova E, Gudipati RK, Lemoine S, Blugeon C, Boulay J, Jensen TH, Stutz F, Devaux F: THO/Sub2p functions to coordinate 3 '-end processing with gene-nuclear pore association. Cell 2008, 135:308-321.

20. Jensen TH, Boulay J, Olesen JR, Colin J, Weyler M, Libri D: Modulation of transcription affects mRNP quality. Mol Cell 2004, 16:235-244.

21. Jimeno S, García-Rubio M, Luna R, Aguilera A: A reduction in RNA polymerase II initiation rate suppresses hyper-recombination and transcription-elongation impairment of THO mutants. Mol Genet Genomics 2008, 280:327-336.

22. Li Y, Wang $X$, Zhang $X$, Goodrich DW: Human hHpr1/p84/Thoc1 regulates transcriptional elongation and physically links RNA polymerase II and RNA processing factors. Mol Cell Biol 2005, 25:4023-4033.

23. Cheng H, Dufu K, Lee C-S, Hsu JL, Dias A, Reed R: Human mRNA export machinery recruited to the 5' End of mRNA. Cell 2006, 127:1389-1400.

24. Katahira J, Inoue H, Hurt YY: Adaptor Aly and co-adaptor Thoc5 function in the Tap-p15-mediated nuclear export of HSP70 mRNA. EMBO J 2009, 28:556-567.

25. El Bounkari O, Guria A, Klebba-Faerber S, Claußen M, Pieler T, Griffiths JR, Whetton AD, Koch A, Tamura T: Nuclear localization of the pre-mRNA associating protein $\mathrm{THOC7}$ depends upon its direct interaction with Fms tyrosine kinase interacting protein (FMIP). FEBS Lett 2009, 583:13-18.

26. Gasparri F, Sola F, Locatelli G, Muzio M: The death domain protein p84N5, but not the short isoform p84N5s, is cell cycle-regulated and shuttles between the nucleus and the cytoplasm. FEBS Lett 2004, 574:13-19.

27. Dias AP, Dufu K, Lei $H$, Reed R: A role for TREX components in the release of spliced mRNA from nuclear speckle domains. Nat Comm 2010, 1:97.

28. Durfee $T$, Mancini MA, Jones D, Elledge SJ, Lee WH: The amino-terminal region of the retinoblastoma gene product binds a novel nuclear matrix protein that co-localizes to centers for RNA processing. J Cell Biol 1994, 127:609-622.

29. Chi B, Wang Q, Wu G, Tan M, Wang L, Shi M, Chang X, Cheng H: Aly and THO are required for assembly of the human TREX complex and association of TREX components with the spliced mRNA. Nucleic Acids Res 2013, 41:1294-1306.

30. Poulton CJ, Schot R, Kia SK, Jones M, Verheijen FW, Venselaar H, deáWit MCY, deáGraaff E, Bertoli-Avella AM, Mancini GMS: Microcephaly with simplified gyration, epilepsy, and infantile diabetes linked to inappropriate apoptosis of neural progenitors. Am J Hum Genet 2011, 89:265-276.

31. Roth KA, D'Sa C: Apoptosis and brain development. Ment Retard Dev D R 2001, 7:261-266

32. O'Driscoll M, Jeggo PA: The role of the DNA damage response pathways in brain development and microcephaly: insight from human disorders. DNA Repair 2008, 7:1039-1050.

33. Li Y, Lin AW, Zhang X, Wang Y, Wang X, Goodrich DW: Cancer cells and normal cells differ in their requirements for Thoc1. Cancer Res 2007, 67:6657-6664

34. Mancini A, Niemann-Seyde SC, Pankow R, El Bounkari O, Klebba-Färber S, Koch A, Jaworska E, Spooncer E, Gruber AD, Whetton AD, Tamura T: 
THOC5/FMIP, an mRNA export TREX complex protein, is essential for hematopoietic primitive cell survival in vivo. BMC Biol 2010, 8:1.

35. Bhalla K, Luo Y, Buchan T, Beachem MA, Guzauskas GF, Ladd S, Bratcher SJ, Schroer RJ, Balsamo J, DuPont BR: Alterations in CDH15 and KIRREL3 in patients with mild to severe intellectual disability. Am J Hum Genet 2008, 83:703-713.

36. Südhof TC: Neuroligins and neurexins link synaptic function to cognitive disease. Nature 2008, 455:903-911.

37. Ropers HH: Genetics of early onset cognitive impairment. Ann Rev of Genomics Hum Genet 2010, 11:161-187.

38. Ramakers GJA: Rho proteins, mental retardation and the cellular basis of cognition. Trends Neurosci 2002, 25:191-199.

39. Mir A, Kaufman L, Noor A, Motazacker MM, Jamil T, Azam M, Kahrizi K, Rafiq MA, Weksberg R, Nasr T: Identification of mutations in TRAPPC9, which encodes the NIK-and IKK-[beta]-binding protein, in nonsyndromic autosomal-recessive mental retardation. Am J Hum Genet 2009, 85:909-915.

40. Mochida GH, Mahainah M, Hill AD, Basel-Vanagaite L, Gleason D, Hill RS, Bodell A, Crosier M, Straussberg R, Walsh CA: A truncating mutation of TRAPPC9 is associated with autosomal-recessive intellectual disability and postnatal microcephaly. Am J Hum Genet 2009, 85:897-902.

41. Philippe O, Rio M, Carioux A, Plaza JM, Guique P, Molinari F, Boddaert N, Bole-Feysot C, Nitschke P, Smahi A: Combination of linkage mapping and microarray-expression analysis identifies NF-[kappa] B signaling defect as a cause of autosomal-recessive mental retardation. Am J Hum Genet 2009, 85:903-908

42. Kaindl AM, Passemard S, Kumar P, Kraemer N, Issa L, Zwirner A: Many roads lead to primary autosomal recessive microcephaly. Prog Neurobiol 2010, 90:363-383.

43. Griffith E, Walker S, Martin CA, Vagnarelli P, Stiff T, Vernay B, Al Sanna N, Saggar A, Hamel B, Earnshaw WC: Mutations in pericentrin cause Seckel syndrome with defective ATR-dependent DNA damage signaling. Nat Genetics 2007, 40:232-236.

44. Raymond F: Monogenic causes of mental retardation. Monogr Hum Genet 2010, 18:89-100.

45. Pak CH, Garshasbi M, Kahrizi K, Gross C, Apponi LH, Noto JJ, Kelly SM, Leung SW, Tzschach A, Behjati F: Mutation of the conserved polyadenosine RNA binding protein, $\mathrm{ZC} 3 \mathrm{H} 14 / \mathrm{dNab2}$, impairs neural function in drosophila and humans. PNAS 2011, 108:12390-12395.

doi:10.1186/1750-1172-8-62

Cite this article as: Beaulieu et al: Intellectual disability associated with a homozygous missense mutation in THOC6. Orphanet Journal of Rare Diseases 2013 8:62

\section{Submit your next manuscript to BioMed Central and take full advantage of:}

- Convenient online submission

- Thorough peer review

- No space constraints or color figure charges

- Immediate publication on acceptance

- Inclusion in PubMed, CAS, Scopus and Google Scholar

- Research which is freely available for redistribution 